\title{
Agomelatine efficacy and acceptability revisited: systematic review and meta-analysis of published and unpublished randomised trials
}

\author{
Markus Koesters, Giuseppe Guaiana, Andrea Cipriani, Thomas Becker and Corrado Barbui
}

\section{Background}

Agomelatine is a novel antidepressant drug with narrative, non-systematic reviews making claims of efficacy.

\section{Aims}

The present study systematically reviewed published and unpublished evidence of the acute and long-term efficacy and acceptability of agomelatine compared with placebo in the treatment of major depression.

\section{Method}

Randomised controlled trials comparing agomelatine with placebo in the treatment of unipolar major depression were systematically reviewed. Primary outcomes were (a) Hamilton Rating Scale for Depression (HRSD) score at the end of treatment (short-term studies) and (b) number of relapses (long-term studies).

\section{Results}

Meta-analyses included 10 acute-phase and 3 relapse prevention studies. Seven of the included studies were unpublished. Acute treatment with agomelatine was associated with a statistically significant superiority over placebo of -1.51 HRSD points $(99 \% \mathrm{Cl}-2.29$ to -0.73 , nine studies). Data extracted from three relapse prevention studies failed to show significant effects of agomelatine over placebo (relative risk $0.78,99 \% \mathrm{Cl} 0.41-1.48$ ). Secondary efficacy analyses showed a significant advantage of agomelatine over placebo in terms of response (with no effect for remission). None of the negative trials were published and conflicting results between published and unpublished studies were observed.

\section{Conclusions}

We found evidence suggesting that a clinically important difference between agomelatine and placebo in patients with unipolar major depression is unlikely. There was evidence of substantial publication bias.

\section{Declaration of interest}

T.B. reports research funding (unrestricted research grants) to the Department of Psychiatry II, UIm University, for clinical trials and investigator-initiated trials from AOK Bundesverband (Berlin), AstraZeneca (Wedel), DGPPN (Deutsche Gesellschaft für Psychiatrie, Psychotherapie und Nervenheilkunde, Berlin), and Robert-Bosch-Stiftung (Stuttgart). The department has also received funds to a minor extent for symposia and in-house training from Affectis (Martinsried), AstraZeneca (Wedel), Bristol-Myers Squibb (München), Euroimmun AG, Fresenius Kabi (Bad Homburg), Janssen Cilag (Neuss), Lilly (Bad Homburg), Lundbeck (Hamburg), PreventPharma GmbH, Servier (München).
Agomelatine is an antidepressant drug with a novel mechanism of action. It is the first antidepressant that targets the circadian system and mediates its therapeutic effect through the melatonergic system. It was approved for the treatment of unipolar major depression by the European Medicines Agency (EMA) in 2009 and has been the focus of several narrative review articles ${ }^{1-6}$ describing its mechanism of action. Despite mainly focusing on the pharmacology of agomelatine, these narrative reviews extensively presented clinical trial data on efficacy and safety, making claims of efficacy in favour of agomelatine. One review ${ }^{4}$ was followed by a still ongoing debate regarding narrative $v$. systematic approaches to evidence synthesis, ${ }^{7}$ and the true clinical efficacy of agomelatine. $^{8-14} \mathrm{~A}$ more critical narrative systematic review was published by Howland, ${ }^{15}$ who concluded that agomelatine has 'a statistical significant, but clinically marginally relevant, antidepressant effect compared with placebo'. There is further evidence of agomelatine efficacy from a meta-analysis of published trials ${ }^{16}$ and from a pooled analysis of selected trials; ${ }^{17}$ however, these reviews failed to evaluate agomelatine efficacy on the basis of a comprehensive systematic review of published and unpublished trials. This is a crucial issue in antidepressant trials, as several studies ${ }^{18-20}$ have illustrated the strong influence of publication bias on the overall estimates of treatment effect, and this may similarly apply to agomelatine trials. ${ }^{21}$ The present systematic review was therefore carried out to assess the evidence of efficacy and acceptability of agomelatine compared with placebo in the acute and long-term treatment of depression using all available evidence (either published or unpublished) and to evaluate the occurrence of publication bias.

\section{Method}

At the beginning of this project, a study protocol was drafted and made freely available to the public on our institutional website before carrying out the final analyses (online supplement DS1). ${ }^{22}$ Furthermore, with the publication of this paper the overall data-set will be in the public domain.

\section{Types of studies and participants}

This systematic review included published and unpublished double-blind, parallel-group, randomised, placebo-controlled trials. We included studies in adult patients ( $>18$ years) with a primary diagnosis of unipolar major depression according to DSM-IV, ${ }^{23}$ DSM-IV-TR ${ }^{24}$ or ICD- $10{ }^{25}$ Studies including patients with a concurrent primary diagnosis of another psychiatric disorder, and studies including participants with depression and with a concomitant medical illness, were excluded.

\section{Types of intervention}

Included trials compared agomelatine with placebo as monotherapy in the acute and relapse prevention treatment of depression. Only treatment arms within the therapeutic dose range of agomelatine 
(25-50 mg/d) were selected. No restrictions regarding pharmaceutical form or dose regimen (fixed or flexible) were applied.

\section{Types of outcome measures}

\section{Primary outcome}

The primary outcome measure for acute-phase studies was the group mean score on the Hamilton Rating Scale for Depression (HRSD) at the end of the trial, or group mean change from baseline to end-point. Despite some criticism, ${ }^{26,27}$ the HRSD is recommended along with the Montgomery-Åsberg Depression Rating Scale (MADRS) by the $\mathrm{EMA}^{28}$ and used in most clinical trials assessing antidepressant efficacy. ${ }^{26,27}$ Clinical interpretation of results from meta-analyses are greatly simplified if effect sizes are calculated as (raw) mean differences. The primary outcome for long-term studies was the proportion of patients who relapsed during the follow-up treatment period. Any definition of depressive relapse according to the study authors was considered.

\section{Secondary outcomes}

For efficacy, we defined the following secondary outcomes.

(a) Group mean scores at the end of the trial, or group mean change from baseline to end-point, on any depression rating scale or Clinical Global Impression Rating scale (CGI). When trials reported results from more than one rating scale, we used the HRSD results or, if not available, the MADRS results. If none of the scales were available, we used the results at any other depression rating scale.

(b) Treatment responders, that is proportion of patients showing a reduction of at least $50 \%$ on the HRSD or MADRS or any other depression scale (for example the Beck Depression Inventory or the Center for Epidemiologic Studies Depression Scale (CES-D)); or who were 'much or very much improved' (score 1 or 2) on the CGI - Improvement scale (CGI-I) or the proportion of patients who improved using any other pre-specified criterion.

(c) Treatment remitters, that is the proportion of patients with a score of seven or less on the 17-item HRSD, or eight or less on the longer versions of the HRSD; ten or less on the MADRS; 'not ill or borderline mentally ill' on the CGI - Severity scale (CGI-S); or any other equivalent value on a depression scale defined by the authors. Preference was given to remission rates defined by HRSD or MADRS scores.

For acceptability, we defined the following secondary outcomes.

(a) Total number of participants who dropped out during the trial as a proportion of the total number of randomised participants: total drop-out rate.

(b) Number of participants who dropped out as a result of inefficacy during the trial as a proportion of the total number of randomised participants.

(c) Number of participants who dropped out as a result of adverse events during the trial as a proportion of the total number of randomised participants.

(d) Total number of participants experiencing adverse events.

\section{Search methods for identification of studies}

Literature searches were performed using the following databases (last update: February 2012): MEDLINE, CINAHL, EMBASE, PsycInfo, Cochrane Central Register of Controlled Trials (CENTRAL). Controlled vocabulary was utilised where appropriate terms were available, supplemented with keyword searches to ensure accurate and exhaustive results. Language or year limits were not applied to any search (online supplement DS2). In order to include unpublished studies, within the time frame of the electronic searches additional hand searches were performed on websites of pharmaceutical companies, clinical trials repositories and registers and regulatory agencies (online supplement DS2).

\section{Data collection}

\section{Selection of studies}

Included and excluded studies were collected following the Preferred Reporting Items for Systematic reviews and MetaAnalyses (PRISMA). ${ }^{29}$ We examined all titles and abstracts, and obtained full texts of potentially relevant papers. Working independently and in duplicate, two reviewers read the papers and determined whether they met inclusion criteria. Considerable care was taken to exclude duplicate publications.

\section{Data extraction and management}

Two review authors, using an electronic data extraction form (EpiData EntryClient v1.1.1.1 for Windows), independently extracted the data on participant characteristics, intervention details and outcome measures. Disagreements were resolved by discussion and consensus with a third member of the team. For continuous outcomes, the mean scores at end-point or the mean change from baseline to end-point, the standard deviation or standard error of these values, and the number of patients included in these analyses, were extracted. ${ }^{30}$ For dichotomous outcomes, the number of patients undergoing the randomisation procedure, the number of patients rated as having responded, remitted or relapsed and the number of patients leaving the study early were recorded.

\section{Assessment of risk of bias in included studies}

The Cochrane risk-of-bias tool ${ }^{31}$ was used. This instrument consists of six items. Two of the items assess the strength of the randomisation process in preventing selection bias in the assignment of participants to interventions, adequacy of sequence generation and allocation concealment. The third item assesses the influence of performance bias on the study results. The fourth item assesses the likelihood of incomplete outcome data, which raise the possibility of bias in effect estimates. The fifth item assesses selective reporting, the tendency to preferentially report statistically significant outcomes. This item requires a comparison of published data with trial protocols, when these are available. The final item refers to other sources of bias (for example sponsorship bias).

\section{Summary statistics}

A double-entry procedure was employed. Data were initially entered and analysed using the Cochrane Collaboration's Review Manager software version 5.1 for Windows, ${ }^{32}$ and subsequently entered into a spreadsheet and re-analysed using the 'metafor' package. ${ }^{33}$ Outputs were cross-checked for internal consistency. When outcome data were not reported, trial authors were asked to supply the data.

\section{Continuous data}

The primary outcome data (acute treatment studies) were analysed by calculating the overall mean differences of studies that used the HRSD. As a secondary outcome, data were analysed using 
standardised mean differences (SMD), as this measure of treatment effect allows the combining of scores from different depression scales. If end-point data were unavailable, change score data were analysed. Where intention-to-treat (ITT) data were available, these were preferred over 'per-protocol analysis'. When only $P$ or standard error values were reported, standard deviations were calculated.

\section{Dichotomous outcomes}

For dichotomous outcomes a Mantel-Haenszel risk ratio was calculated. Response, remission and relapse rates were calculated using an ITT analysis: if participants left the study before the intended end-point, it was assumed that they had experienced the negative outcome. In case of missing information, we estimated the number of patients responding to treatment using a validated imputation method. ${ }^{34,35}$ The robustness of this approach was checked in a sensitivity analysis.

\section{Confidence intervals}

A $99 \%$ confidence interval was calculated for all efficacy estimates according to Barbui and colleagues. ${ }^{36}$ This approach, instead of a $95 \%$ confidence interval approach, was adopted to have the widest estimate of likely true effect. We set the level of significance at 0.01 as we made multiple comparisons and reasoned that only robust differences between treatments should inform clinical practice. In fact, it is more important to avoid the possibility of showing a difference in the absence of a true difference, than to avoid the possibility of not showing a difference in the presence of a true difference. In other words, we gave priority to avoiding a type I rather than a type II error. ${ }^{37}$ Conversely, a $95 \%$ confidence interval was calculated for all tolerability estimates. In terms of tolerability it is more important to avoid the possibility of not showing a difference in the presence of a true difference than to avoid the possibility of showing a difference in the absence of a true difference. In other words, we gave priority to avoiding a type II rather than a type I error.

\section{Studies with multiple treatment groups}

For dichotomous outcomes, trials comparing different doses of agomelatine with placebo were converted into two-arm trials by summing samples and averaging doses. For continuous outcomes, means and standard deviations of different dosage arms were combined into a single arm according to the Cochrane Handbook. ${ }^{38}$

\section{Assessment of heterogeneity}

Visual inspection of graphs was used to investigate the possibility of statistical heterogeneity. This was supplemented using the $I^{2}$ statistic. This provides an estimate of the percentage of variability due to heterogeneity rather than chance alone. Where the $I^{2}$ estimate was greater than or equal to $50 \%$ we interpreted this as indicating the presence of high levels of heterogeneity. ${ }^{39}$ Statistical significance of heterogeneity was additionally tested with $\chi^{2}$-tests, using a threshold of $P<0.20$ as the threshold of statistical significance, because the power of this test is known to be low if the number of studies included is small. ${ }^{39}$

\section{Assessment of publication bias}

For the primary outcome, the funnel plot approach was used to investigate publication bias.

\section{Data synthesis and presentation}

Continuous and dichotomous outcomes were analysed using a random-effects model, as this takes into account any differences between studies even if there is no statistically significant heterogeneity. ${ }^{31}$ A summary of findings table was produced according to the methodology described by the GRADE working group. ${ }^{40,41}$

\section{Subgroup analyses}

The following pre-planned subgroup analyses were carried out: (a) agomelatine dose (low dosage: $25 \mathrm{mg} / \mathrm{d} v$. flexible doses and $50 \mathrm{mg} / \mathrm{d}$ ); (b) publication status (published $v$. unpublished studies); (c) data imputation (file $v$. imputed).

\section{Results}

\section{Characteristics of included studies}

From 130 potentially relevant records from the search of databases and additional sources, 90 were excluded on the basis of title or abstract. The remaining 40 studies were retrieved for more detailed evaluation (Fig. 1). Overall, 13 studies, ${ }^{42-54}$ including 7 unpublished studies, ${ }^{42-46,50,51}$ met the inclusion criteria. Of these, $10^{43-46,48-50,52-54}$ were short-term studies and $3^{42,47,51}$ were long-term relapse prevention studies (see online supplement DS3 for references of the 27 excluded studies). The main characteristics of the 13 studies are reported in Table 1. All comparisons included more than 100 patients per treatment arm, the length of follow-up ranged between 6 and 52 weeks. One study ${ }^{45}$ was carried out in individuals aged 60 or above. None of the studies recruited patients in primary healthcare settings. All studies were financially supported by agomelatine manufacturing companies. The overall quality of reporting was graded as moderate to good (Fig. 2, online supplement DS4, online Fig. DS1 and online Table DS1).

\section{Efficacy of agomelatine}

\section{Primary outcomes}

With the exception of one study, ${ }^{45}$ all studies reported HRSD scores. Acute-phase studies (9 studies, overall 2947 patients) indicated that acute treatment with agomelatine is associated with a statistically significant difference over placebo of -1.51 points on the HRSD (99\% CI -2.29 to -0.73 ) (Fig. 3). The subgroup analysis comparing published $v$. unpublished trials revealed a difference of -1.39 HRSD points $(P=0.02)$ between unpublished (mean difference: $-0.73,99 \% \mathrm{CI}-1.90$ to 0.45 ) and published trials (mean difference: $-2.12,99 \%$ CI -3.16 to -1.08 , Fig. $3)$. In terms of risk of relapse, data extracted from three long-term studies (overall 983 patients) failed to show any significant effect of agomelatine over placebo (relative risk $=0.78,99 \%$ CI $0.41-1.48$, Fig. 4).

\section{Secondary outcomes}

The analysis of secondary outcomes is summarised in Table 2 and fully reported in online Figs DS2-7. In terms of response the data extracted from the 10 acute-phase studies (overall 3295 patients) showed a significant advantage of agomelatine over placebo (online Fig. DS2), whereas in terms of remission (7 studies, overall 2346 patients) no difference was found between agomelatine and placebo (online Fig. DS3). Both analyses showed a statistically significant effect of agomelatine in the subgroup of published trials only.

In the analysis of depressive symptoms where scores from different rating scales were pooled, data extracted from the 10 acute-phase studies (2896 patients) indicated that acute treatment 


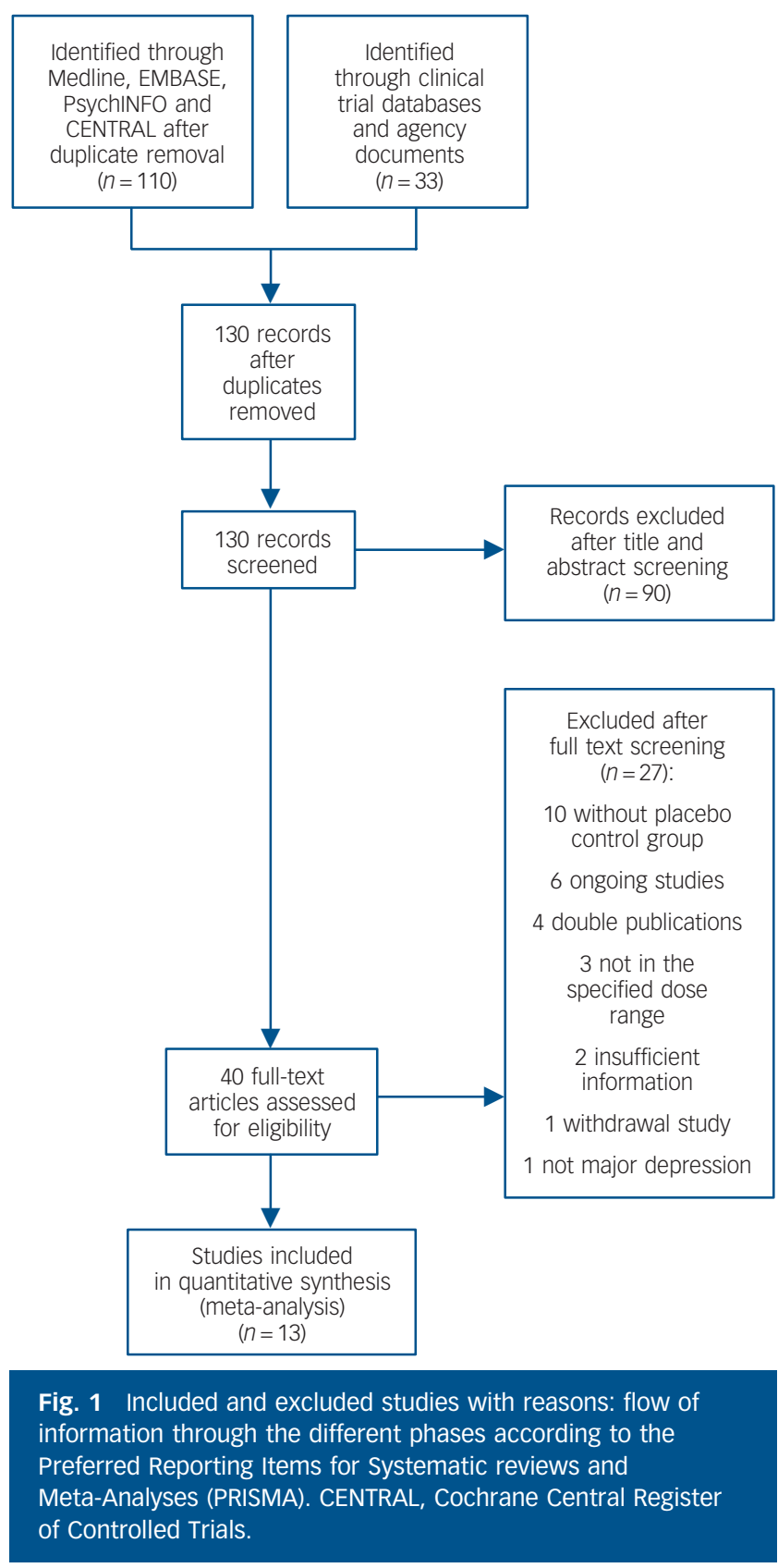

with agomelatine is associated with an SMD over placebo of -0.18 (99\% CI -0.27 to -0.08 ) (Table 2, online Fig. DS4). When studies were grouped into published $v$. unpublished, a significant antidepressant effect of agomelatine was shown in the subgroup of published trials only.

\section{Acceptability and tolerability of agomelatine}

In terms of overall acceptability, data extracted from 9 acute-phase studies (1782 patients treated with agomelatine and 1313 with placebo) failed to show a significant difference between agomelatine and placebo (Fig. 5). Whereas discontinuation because of inefficacy significantly favoured agomelatine (online Fig. DS5), discontinuation because of adverse events did not differ between agomelatine and placebo (Table 2 and online Fig. DS6). Similarly, no difference emerged from the analysis of the proportion of patients reporting adverse events (Table 2 and online Fig. DS7).

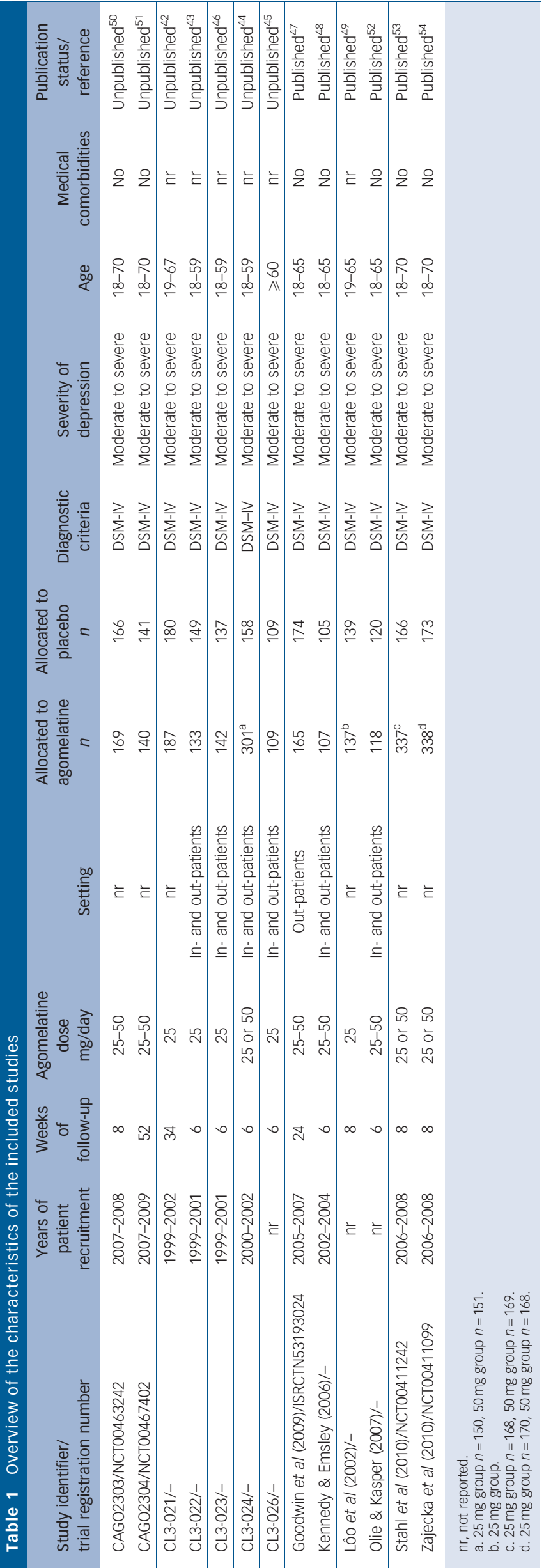


Random sequence generation (selection bias)

Allocation concealment (selection bias)

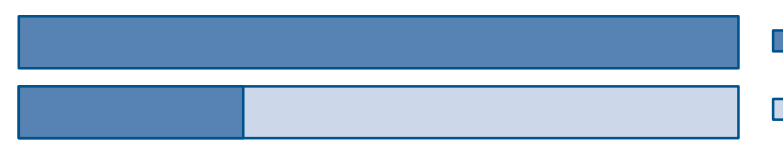

Masking of participants and personnel (performance bias)

Masking of outcome assessment (detection bias)

Incomplete outcome data (attrition bias)

Selective reporting (reporting bias)

Other bias
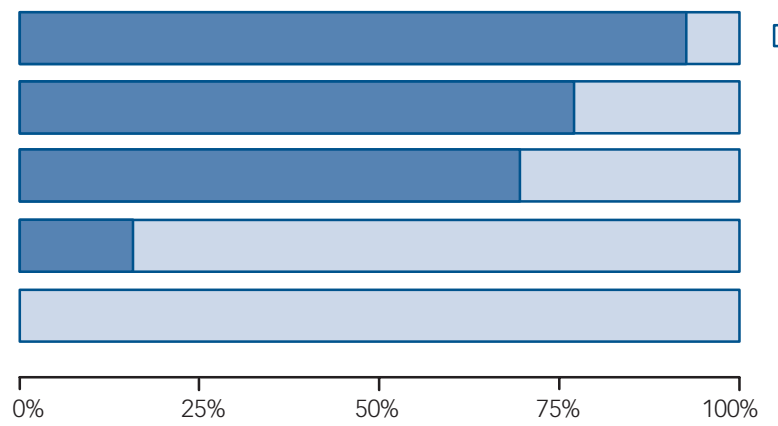

Fig. 2 Methodological quality graph: risk of bias item presented as percentage of studies with low, unclear or high risk of bias.

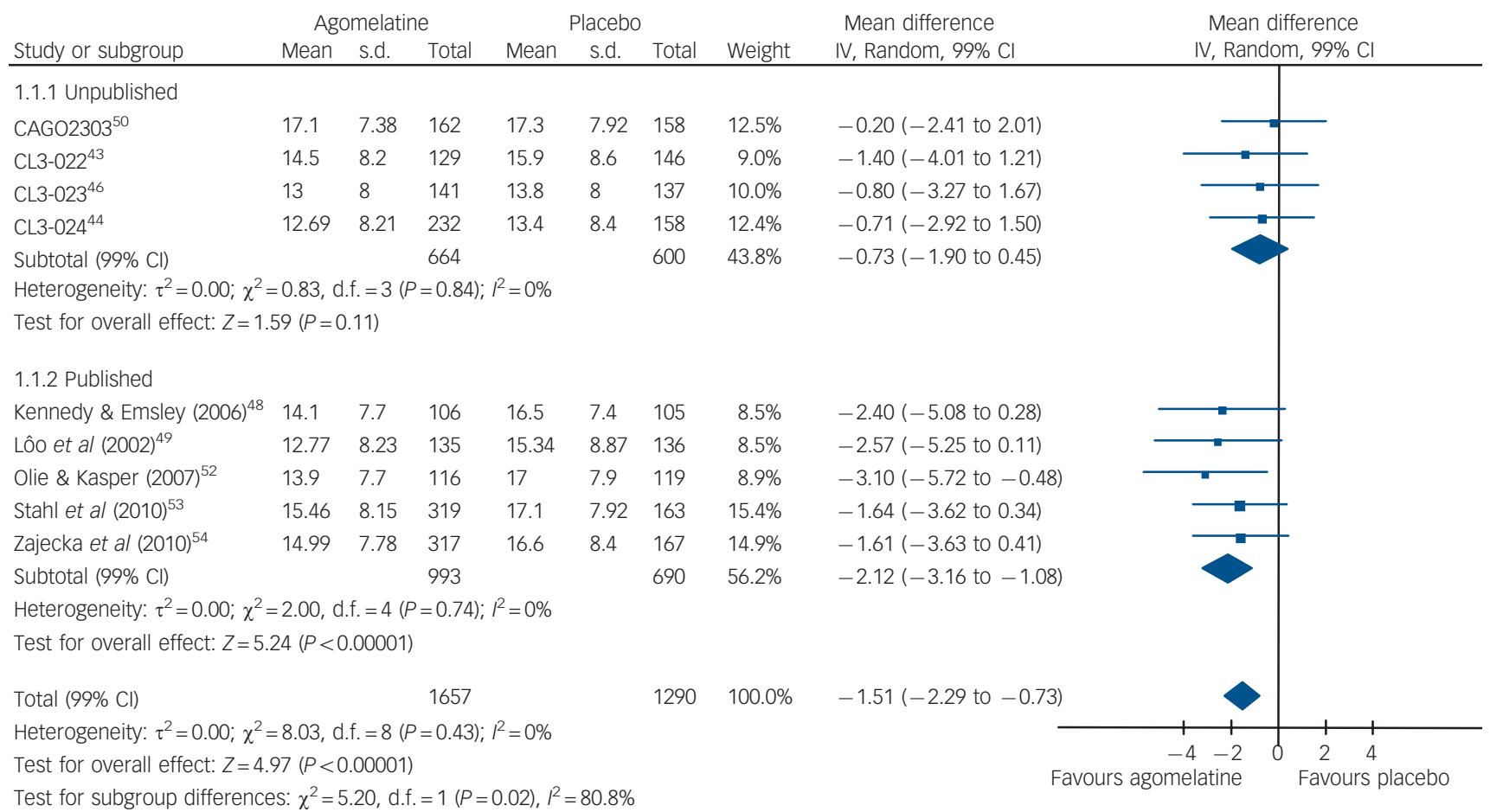

Fig. 3 Random-effects meta-analysis of the effect of agomelatine v. placebo on the Hamilton Rating Scale for Depression scores.

\section{Agomelatine dose}

Data extracted from studies that employed a fixed dose of $25 \mathrm{mg} / \mathrm{d}$ of agomelatine (mean difference: $-1.47,99 \%$ CI -2.50 to $-0.44,6$ studies, 1696 participants) provided a similar overall estimate compared with studies that employed a flexible dose scheme of $25-50 \mathrm{mg} / \mathrm{d}$ or a fixed dose of $50 \mathrm{mg} / \mathrm{d}$ (mean difference: $-1.57,99 \%$ CI -2.90 to $-0.24,6$ studies, 1644 participants) (online Fig. DS8).

\section{Data imputation and publication bias}

The exclusion of studies with imputed data did not change the overall findings (online Fig. DS9). The funnel plot did not suggest the occurrence of publication bias, although it clearly showed that the omission of the unpublished trials that we were able to include would have led to a biased overall estimate (online Fig. DS10).

\section{Discussion}

\section{Main findings}

The present systematic review found that acute treatment with agomelatine is associated with a difference of 1.5 points on the HRSD. This difference was statistically significant, although the clinical relevance of this small effect is questionable. No research evidence or consensus is available about what constitutes a clinically meaningful difference in HRSD scores. Antidepressant research has recently faced the issues of (a) a large number of studies reporting negative findings and (b) a possible increase in placebo response rates, which may be caused by changes in selection of study participants and how studies are conducted. ${ }^{55}$ Such changes might contribute to a reduction in the likelihood of identifying drug effectiveness in antidepressant drug trials. 


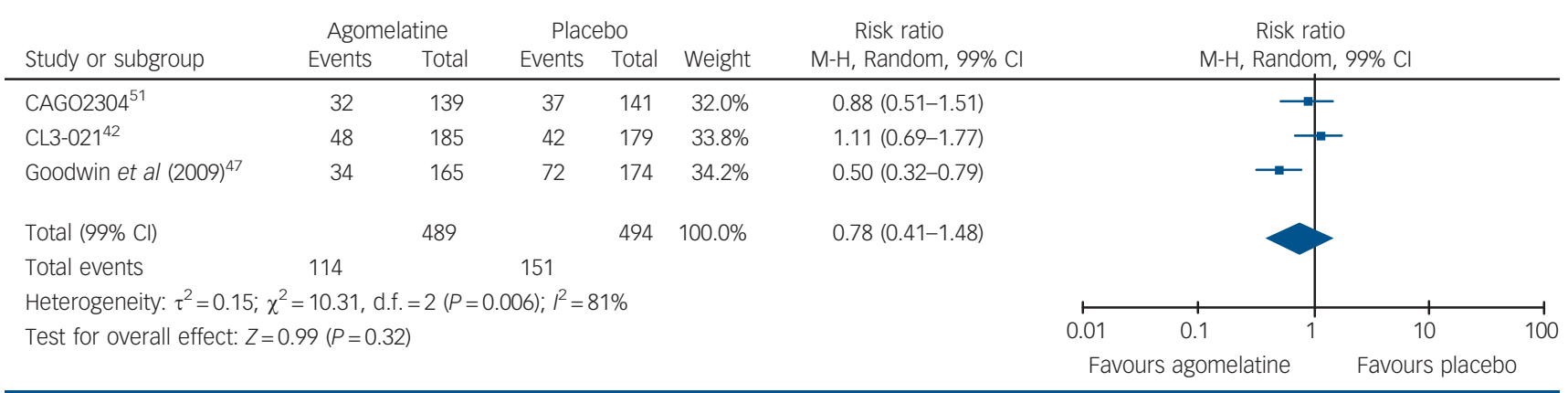

Fig. 4 Random-effects meta-analysis of the effect of agomelatine $v$. placebo on the risk of relapse in long-term studies.

\begin{tabular}{|c|c|c|c|c|c|c|}
\hline & Studies & $\begin{array}{c}\text { Agomelatine } \\
n / N\end{array}$ & $\begin{array}{c}\text { Placebo } \\
n / N\end{array}$ & Effect size (Cl) & Measure & $I^{2}, \%$ \\
\hline Failure to respond & 10 & $971 / 1878$ & $830 / 1417$ & 0.87 (0.80 to 0.94$)$ & RR $(99 \%$ Cl) & 0 \\
\hline Failure to show remission & 7 & 1098/1333 & $867 / 1013$ & 0.97 (0.89 to 1.05$)$ & RR $(99 \% \mathrm{Cl})$ & 70 \\
\hline Depressive symptoms & 10 & 1763 & 1133 & $-0.18(-0.27$ to -0.08$)$ & SMD $(99 \% \mathrm{Cl})$ & 2 \\
\hline Discontinuation due to inefficacy & 8 & $58 / 1481$ & $88 / 1155$ & 0.60 (0.43 to 0.83$)$ & $\mathrm{RR}(95 \% \mathrm{Cl})$ & 0 \\
\hline Discontinuation due to adverse events & 8 & $65 / 1481$ & $54 / 1155$ & 0.90 (0.63 to 1.30$)$ & $\mathrm{RR}(95 \% \mathrm{Cl})$ & 0 \\
\hline Any adverse event & 6 & $777 / 1188$ & $557 / 861$ & 0.98 (0.92 to 1.04$)$ & RR $(95 \% \mathrm{Cl})$ & 0 \\
\hline \multicolumn{7}{|l|}{$\begin{array}{l}\text { RR, risk ratio; SMD, standardised mean difference. } \\
\text { a. See online Figs DS2-7. }\end{array}$} \\
\hline
\end{tabular}

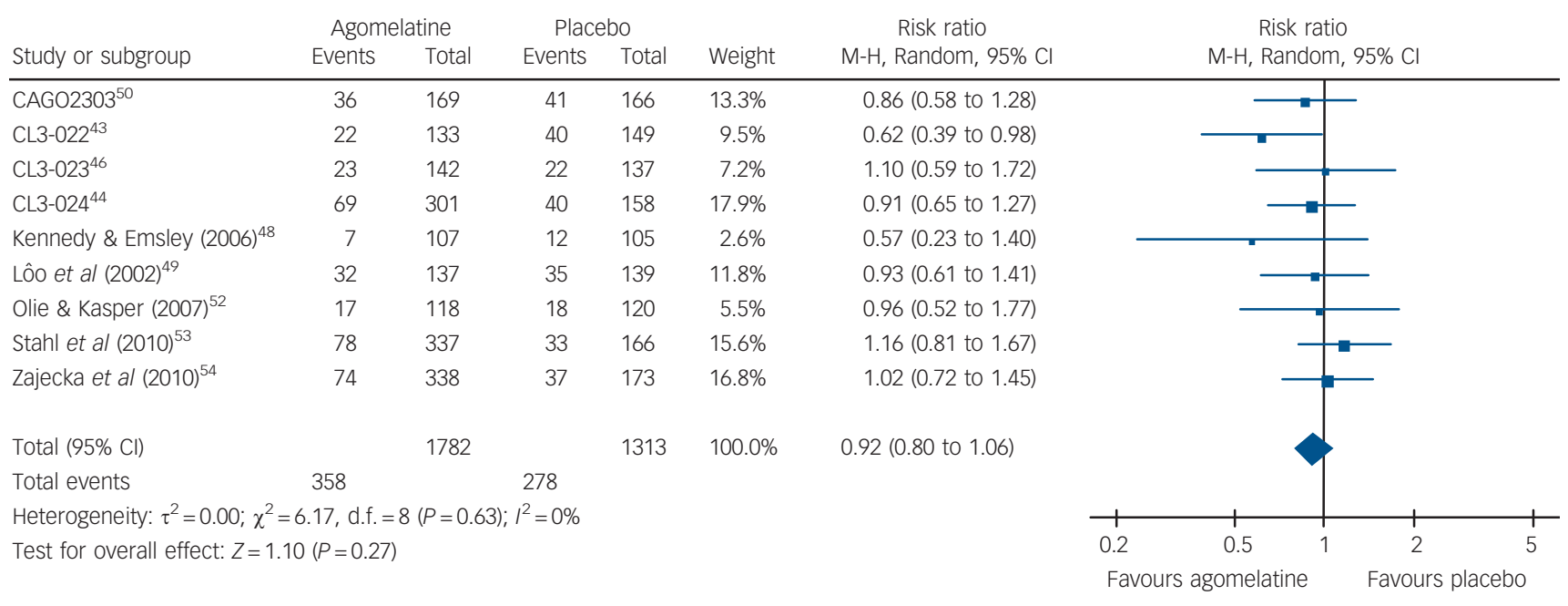

Fig. 5 Random-effects meta-analysis of the effect of agomelatine $v$. placebo on treatment discontinuation by any cause.

However, even with this consideration in mind, it is plausible to agree with one of the agomelatine clinical trials ${ }^{53}$ that a difference of less than three HRSD points is unlikely to be clinically meaningful. Other publications have discussed a difference of two points as being clinically important, ${ }^{2,56}$ but the effect of agomelatine in our review was also below this threshold. Furthermore, it cannot be excluded that a 1.5-point difference may reflect a weak effect on sleep- regulating mechanisms rather than a genuine antidepressant effect.

In a recent statement, the EMA Committee for Medicinal Products for Human Use (CHMP) pointed out that, in addition to a statistically significant effect in symptom scale scores, the clinical relevance has to be confirmed by responder and remitter analyses and that '.. . results in the short-term trials need to be confirmed in clinical trials, to demonstrate the maintenance of effects. ${ }^{57}$ For dichotomous outcomes, agomelatine was not superior to placebo in terms of relapse and remission rates, but was statistically superior to placebo in terms of response rates. The difference in response rates corresponds to an absolute risk difference of $6 \%$ and to a number needed to treat (NNT) of 15 . Based on an analysis of regulatory submissions, ${ }^{56}$ which found an average difference of $16 \%$ in the response rates between common antidepressants and placebo, EMA CHMP states that this difference '. . . is considered to be the lower limit of the pharmacological effect that would be expected in clinical practice. $^{57}$ Other authors ${ }^{2}$ considered an NNT of ten or below as clinically relevant. Clearly, the effect size in the present analysis is of doubtful clinical significance. This point is strengthened by 
the fact that depression is a clinical condition for which many active antidepressants are already available. ${ }^{58}$

Doubt emerged regarding the value of agomelatine as a firstline agent when Novartis dropped the agomelatine development programme, ${ }^{59}$ and the current manufacturer has informed the UK National Institute for Health and Care Excellence (NICE) that it would not be making an evidence submission for the appraisal of agomelatine for the treatment of major depressive episodes. ${ }^{58}$ Based on the results presented in this review, we suggest that agomelatine should not be used as a first-line treatment in patients with major depression.

\section{Problems with current methods for approving new drugs}

In Europe new drugs are approved or rejected on the basis of the results of studies carried out by the manufacturer and submitted to the EMA. We note that decisions are taken on the basis of the results of individual studies with no role for aggregating efficacy data using meta-analytic techniques. We argue, however, that pooling studies would have some beneficial consequences for the review process by increasing statistical power and by contributing to the detection of between-study heterogeneity.

Additionally, the EMA should require submission of all available studies, without allowing manufacturers to submit only a selection of clinical trials. For example, in the agomelatine European Assessment Report (EPAR) two of the three long-term studies are included, and these two studies were assessed using a questionable narrative approach. The EMA reports: 'in the first of the long-term studies, there was no difference between Valdoxan and placebo in preventing symptoms returning during 26 weeks of treatment. However, the second study showed that symptoms returned in $21 \%$ of the patients taking Valdoxan over 24 weeks (34 out of 165), compared with $41 \%$ of the patients taking placebo (72 out of 174$)^{\prime} .^{60}$

The present systematic review showed that pooling the three long-term studies failed to show a difference between agomelatine and placebo in terms of relapse rate, but even if the two studies included in the EMA assessment had been pooled, lack of agomelatine efficacy would have been found. According to the earlier-mentioned CHMP statement, ${ }^{57}$ the lack of long-term effects further limits the clinical relevance of the effect of agomelatine. We argue that the EMA qualitative approach should be assisted by a quantitative approach to data synthesis.

\section{Limitations}

This systematic review has some limitations. Although we were able to include several unpublished studies, uncertainty remains as to whether other randomised studies have been carried out and never published or disseminated on the World Wide Web. This is a major issue as we have demonstrated that the results of unpublished agomelatine studies were systematically less favourable in comparison with published ones. It is therefore possible that the true effect of agomelatine might be smaller than the effect we calculated in the present systematic review. Also, comparative head-to-head studies were not included. According to the study protocol, the aim of the present study was to assess efficacy and acceptability of agomelatine combining both published and unpublished placebo-controlled trials. We acknowledge that comparative effectiveness is of paramount relevance in a field characterised by many active treatments, but we reasoned that a standard direct meta-analysis was not the most suitable methodological approach. When a new drug is compared with several other treatments, more sophisticated approaches integrating direct and indirect evidence have been developed and already applied to antidepressants. ${ }^{35}$ It is likely that the introduction of agomelatine in these network analyses with other antidepressants and placebos will shed light on the relative value of this new drug.

To analyse efficacy we employed a $99 \%$ confidence interval. This is not the standard way to assess efficacy, as usually a $95 \%$ confidence interval is preferred in all outcomes. However, we decided a priori to apply this method because it has been used previously in the field of placebo-controlled antidepressant trials and shown to be the most clinically informative. ${ }^{36,37}$

The extent of publication bias found in the present review was surprising. None of the negative trials were published, and the standardised effect size was more than three times higher in published than in unpublished trials. However, Singh and colleagues $^{16}$ also questioned the clinical relevance of the antidepressant effect of agomelatine, although their meta-analysis included published trials only. Publication bias was even more obvious in relapse prevention trials. There is only one positive trial published suggesting that agomelatine is effective in relapse prevention. The present review included unpublished data from 1908 patients and published data on 2022 patients randomised to short- or long-term trials of agomelatine. Thus, only about $50 \%$ of the data available was published. Furthermore, only the minority of studies included were registered in public trial databases. This indicates that clinical trial registration in its current form has not yet solved the issue of publication and outcome reporting bias. As has already been suggested, ${ }^{61}$ better access to clinical trial data would make them more useful for doctors, researchers and consumers.

Markus Koesters, Department of Psychiatry II, UIm University, UIm, Germany; Giuseppe Guaiana, Department of Psychiatry, University of Western Ontario, St Thomas, Canada; Andrea Cipriani, Department of Public Health and Community Medicine, Section of Psychiatry, University of Verona, Verona, Italy; Thomas Becker, Department of Psychiatry II, UIm University, UIm, Germany; Corrado Barbui, Department of Public Health and Community Medicine, Section of Psychiatry, University of Verona, Verona, Italy

Correspondence: Markus Kösters, Department of Psychiatry II, UIm University, Ulm, Ludwig-Heilmeyer-Str. 2, 89312 Guenzburg, Germany. Email: Markus.Koesters@uni-ulm.de

First received 12 Sep 2012, final revision 5 Mar 2013, accepted 4 Apr 2013

\section{Acknowledgements}

We thank Katja Häderle and Lena Staudigl for their help with the literature and data management.

\section{References}

1 Arendt J, Rajaratnam SMW. Melatonin and its agonists: an update. $\mathrm{Br} J$ Psychiatry 2008; 193: 267-9.

2 Montgomery SA, Moller $\mathrm{HJ}$. Is the significant superiority of escitalopram compared with other antidepressants clinically relevant? Int Clin Psychopharmacol 2009; 24: 111-8.

3 de Bodinat C, Guardiola-Lemaitre B, Mocaer E, Renard P, Munoz C, Millan MJ. Agomelatine, the first melatonergic antidepressant: discovery, characterization and development. Nat Rev Drug Discov 2010; 9: 628-42.

4 Hickie IB, Rogers NL. Novel melatonin-based therapies: potential advances in the treatment of major depression. Lancet 2011; 378: 621-31.

5 Lam RW. The importance of early symptom relief in antidepressant treatment: focus on agomelatine. J Psychopharmacol 2010; 24: 27-30.

6 Llorca PM. The antidepressant agomelatine improves the quality of life of depressed patients: implications for remission. J Psychopharmacol 2010; 24: 21-6.

7 Barbui C, Cipriani A. Agomelatine and the brave old world of narrative-based medicine. Evid Based Ment Health 2012; 15: 2-3. 
8 Barbui C, Cipriani A. Novel melatonin-based treatments for major depression. Lancet 2012; 379: 215.

9 Carroll BJ. Novel melatonin-based treatments for major depression. Lancet 2012; 379: 216

10 Howland RH. Novel melatonin-based treatments for major depression. Lancet 2012; 379: 215-6.

11 Jureidini J, Raven M. Novel melatonin-based treatments for major depression. Lancet 2012; 379: 216-7.

12 Lloret-Linares C, Bergmann JF, Mouly S. Novel melatonin-based treatments for major depression. Lancet 2012; 379: 216.

13 Serfaty M, Raven PW. Novel melatonin-based treatments for major depression. Lancet 2012; 379: 217.

14 Hickie IB, Rogers NL. Novel melatonin-based treatments for major depression? Authors' reply. Lancet 2012; 379: 217-9.

15 Howland RH. A benefit-risk assessment of agomelatine in the treatment of major depression. Drug Saf 2011; 34: 709-31.

16 Singh SP, Singh V, Kar N. Efficacy of agomelatine in major depressive disorder: meta-analysis and appraisal. Int J Neuropsychopharmacol 2011; 15 417-28.

17 Montgomery SA, Kasper S. Severe depression and antidepressants: focus on a pooled analysis of placebo-controlled studies on agomelatine. Int Clin Psychopharmacol 2007; 22: 283-91

18 Eyding D, Lelgemann M, Grouven U, Harter M, Kromp M, Kaiser T, et al. Reboxetine for acute treatment of major depression: systematic review and meta-analysis of published and unpublished placebo and selective serotonin reuptake inhibitor controlled trials. BMJ 2010; 341: c4737.

19 Kirsch I, Deacon BJ, Huedo-Medina TB, Scoboria A, MooreTJ, Johnson BT. Initial severity and antidepressant benefits: a meta-analysis of data submitted to the Food and Drug Administration. PLOS Med 2008; 5: e45.

20 Turner EH, Matthews AM, Linardatos E, Tell RA, Rosenthal R. Selective publication of antidepressant trials and its influence on apparent efficacy. N Engl J Med 2008; 358: 252-60.

21 Howland RH. Publication bias and outcome reporting bias: agomelatine as a case example. J Psychosoc Nurs Ment Health Serv 2011; 49: 11-4.

22 Koesters M, Cipriani A, Guaiana G, Becker T, Barbui C. Agomelatine versus Placebo: A Meta-Analysis of Published and Unpublished Trials. Protocol for a Systematic Review. University of Ulm, 2012 (http://vts.uni-ulm.de/ doc. asp? id $=7851$ )

23 American Psychiatric Association. Diagnostic and Statistical Manual of Mental Disorders (4th edn) (DSM-IV). APA, 1994.

24 American Psychiatric Association. Diagnostic and Statistical Manual of Mental Disorders (4th edn, text revision) (DSM-IV-TR). APA, 2000.

25 World Health Organization. The ICD-10 Classification of Mental and Behavioural Disorders: Clinical Descriptions and Diagnostic Guidelines. WHO, 1992.

26 Bagby RM, Ryder AG, Schuller DR, Marshall MB. The Hamilton Depression Rating Scale: has the gold standard become a lead weight? Am J Psychiatry 2004; 161: 2163-77.

27 Isacsson G, Adler M. Randomized clinical trials underestimate the efficacy of antidepressants in less severe depression. Acta Psychiatr Scand 2012; 125 453-9.

28 European Agency for the Evaluation of Medicinal Products. Note for Guidance on Clinical Investigation of Medicinal Products in the Treatment of Depression. European Agency for the Evaluation of Medicinal Products, 2002 (http://www.ema.europa.eu/docs/en_GB/document_library/Scientific_guideline/2009/09/WC500003526.pdf).

29 Moher D, Liberati A, Tetzlaff J, Altman DG. Preferred reporting items for systematic reviews and meta-analyses: the PRISMA statement. BMJ 2009; 339: b2535.

30 Norman GR. Issues in the use of change scores in randomized trials. J Clin Epidemiol 1989; 42: 1097-105.

31 Higgins J, Altman DG, Sterne J. Assessing risk of bias in included studies. In Cochrane Handbook for Systematic Reviews of Interventions Version 5.1.0 (eds J Higgins and S Green). The Cochrane Collaboration, 2011.

32 The Nordic Cochrane Centre, The Cochrane Collaboration. Review Manager (RevMan) (5.1). The Nordic Cochrane Centre, The Cochrane Collaboration, 2011.

33 Viechtbauer W. Conducting meta-analyses in $\mathrm{R}$ with the metafor package. J Stat Softw 2010; 36: 1-48.

34 Furukawa TA, Cipriani A, Barbui C, Brambilla P, Watanabe N. Imputing response rates from means and standard deviations in meta-analyses. Int Clin Psychopharmacol 2005; 20: 49-52.

35 Cipriani A, Furukawa TA, Salanti G, Geddes JR, Higgins JP, Churchill R, et al. Comparative efficacy and acceptability of 12 new-generation antidepressants: a multiple-treatments meta-analysis. Lancet 2009; 373: 746-58.

36 Barbui C, Furukawa TA, Cipriani A. Effectiveness of paroxetine in the treatment of acute major depression in adults: a systematic re-examination of published and unpublished data from randomized trials. CMAJ 2008; 178: 296-305.

37 Cipriani A, Brambilla P, Furukawa T, Geddes J, Gregis M, Hotopf M, et al. Fluoxetine versus other types of pharmacotherapy for depression. Cochrane Database Syst Rev 2005; 4: CD004185.

38 Higgins J, Deeks JJ. Selecting studies and collecting data. In Cochrane Handbook for Systematic Reviews of Interventions Version 5.1.0 (eds J Higgins, S Green). The Cochrane Collaboration, 2011.

39 Higgins JP, Thompson SG, Deeks JJ, Altman DG. Measuring inconsistency in meta-analyses. BMJ 2003; 327: 557-60.

40 Guyatt GH, Oxman AD, Kunz R, Vist GE, Falck-Ytter Y, Schunemann HJ. What is "quality of evidence" and why is it important to clinicians? BMJ 2008; 336: 995-8.

41 Guyatt GH, Oxman AD, Vist GE, Kunz R, Falck-Ytter $Y$, Alonso-Coello $P$, et al. GRADE: an emerging consensus on rating quality of evidence and strength of recommendations. BMJ 2008; 336: 924-6.

42 European Agency for the Evaluation of Medicinal Products. CL3-021. Unpublished study, data from EMA reports. In CHMP Assessment Report for Valdoxan. European Agency for the Evaluation of Medicinal Products, 2008 (http://www.ema.europa.eu/docs/en_GB/document_library/ EPAR_-_Public_assessment_report/human/000915/WC500046226.pdf).

43 European Agency for the Evaluation of Medicinal Products. CL3-022. Unpublished study, data from EMA reports. In CHMP Assessment Report for Valdoxan. European Agency for the Evaluation of Medicinal Products, 2008 (http://www.ema.europa.eu/docs/en_GB/document_library/ EPAR_-_Public_assessment_report/human/000915/WC500046226.pdf).

44 European Agency for the Evaluation of Medicinal Products. CL3-024. Unpublished study, data from EMA reports. In CHMP Assessment Report for Valdoxan. European Agency for the Evaluation of Medicinal Products, 2008 (http://www.ema.europa.eu/docs/en_GB/document_library/ EPAR_-_Public_assessment_report/human/000915/WC500046226.pdf).

45 European Agency for the Evaluation of Medicinal Products. CL3-026. Unpublished study, data from EMA reports. In CHMP Assessment Report for Valdoxan. European Agency for the Evaluation of Medicinal Products, 2008 (http://www.ema.europa.eu/docs/en_GB/document_library/ EPAR_-_Public_assessment_report/human/000915/WC500046226.pdf).

46 European Agency for the Evaluation of Medicinal Products. CL3-023. Unpublished study, data from EMA reports. In CHMP Assessment Report for Valdoxan. European Agency for the Evaluation of Medicinal Products, 2008 (http://www.ema.europa.eu/docs/en_GB/document_library/ EPAR__Public_assessment_report/human/000915/WC500046226.pdf).

47 Goodwin GM, Emsley R, Rembry S, Rouillon F. Agomelatine prevents relapse in patients with major depressive disorder without evidence of a discontinuation syndrome: a 24-week randomized, double-blind, placebo-controlled trial. J Clin Psychiatry 2009; 70: 1128-37.

48 Kennedy SH, Emsley R. Placebo-controlled trial of agomelatine in the treatment of major depressive disorder. Eur Neuropsychopharmacol 2006; 16: 93-100.

49 Lôo H, Hale A, D'haenen H. Determination of the dose of agomelatine, a melatoninergic agonist and selective $5-\mathrm{HT}(2 \mathrm{C})$ antagonist, in the treatment of major depressive disorder: a placebo-controlled dose range study. Int Clin Psychopharmacol 2002; 17: 239-47.

50 Novartis. CAGO178A2303. Unpublished Study. Novartis, 2011 (http:// www.novctrd.com/ctrdWebApp/clinicaltrialrepository/displayFile.do? trialResult = 2659).

51 Novartis. CAG0178A2304. Unpublished Study. Novartis, 2011 (http:// www.novctrd.com/ctrdWebApp/clinicaltrialrepository/displayFile.do? trialResult $=3420$ ).

52 Olie JP, Kasper S. Efficacy of agomelatine, a MT1/MT2 receptor agonist with 5-HT2C antagonistic properties, in major depressive disorder. Int J Neuropsychopharmacol 2007; 10: 661-73.

53 Stahl SM, Fava M, Trivedi MH, Caputo A, Shah A, Post A. Agomelatine in the treatment of major depressive disorder: an 8-week, multicenter, randomized, placebo-controlled trial. J Clin Psychiatry 2010; 71: 616-26.

54 Zajecka J, schatzberg A, Stahl S, Shah A, Caputo A, Post A. Efficacy and safety of agomelatine in the treatment of major depressive disorder: a multicenter, randomized, double-blind, placebo-controlled trial. J Clin Psychopharmacol 2010; 30: 135-44.

55 Undurraga J, Baldessarini RJ. Randomized, placebo-controlled trials of antidepressants for acute major depression: thirty-year meta-analytic review. Neuropsychopharmacology 2012; 37: 851-64. 
56 Melander $\mathrm{H}$, Salmonson $\mathrm{T}$, Abadie $\mathrm{E}$, van Zwieten-Boot B. A regulatory apologia - a review of placebo-controlled studies in regulatory submissions of new-generation antidepressants. Eur Neuropsychopharmacol 2008; 18 : 623-7.

57 Broich K. Committee for Medicinal Products for Human Use (CHMP) assessment on efficacy of antidepressants. Eur Neuropsychopharmacol 2009; 19: 305-8.

58 National Institute for Health and Clinical Excellence. Agomelatine for the Treatment of Major Depressive Episodes (Terminated Appraisal). NICE, 2011 (http://www.nice.org.uk/nicemedia/live/13539/55586/55586.pdf).
59 Novartis. Financial Report Q3 2011. Novartis, 2011 (http://www.novartis com/downloads/investors/financial-results/quarterly-results/q3-2011-mediarelease-en.pdf).

60 European Medicines Agency. What Benefit has Valdoxan Shown During the Studies? European Medicines Agency, 2012 (http://www.ema.europa.eu/ ema/index.jsp?curl = pages/medicines/human/medicines/000915/human med_001123.jsp\&mid = WC0b01ac058001d124\&jsenabled = true).

61 Gøtzsche PC. Why we need easy access to all data from all clinical trials and how to accomplish it. Trials 2011; 12: 249.

\section{Frantz Fanon's Black Skin, White Masks}

\section{Roland Littlewood}

Fanon's Peau Noire, Masques Blancs, the 1952 revision of his (rejected) doctoral thesis, strikes the native English speaker as unduly epigrammatic and poetic, yet its impassioned denunciation of racism and colonialism is replete with citations of Sartre and Merleau-Ponty, Hegel and Marx, Jaspers and Anna Freud.

Fanon was born in 1928 into a comfortable middle-class Creole family in the West Indian island of Martinique, then a French colony and now a département of France. Fighting for the Free French forces in the Second World War, afterwards he proceeded to Lyons to study medicine. Appointed in 1953 as a consultant psychiatrist in French Algeria, then about to commence its war of liberation from the metropolis, Fanon supported the Algerian resistance while continuing to practise medically, and wrote articles on the effects of torture and atrocity on the local civilian population, but also on the French military. He resigned from his post to join the rebel F.L.N. headquarters in Tunis, and in 1960 was appointed ambassador from the provisional Algerian government to Nkrumah's Ghana. He died of leukaemia the following year.

The 1952 book had taken a more restricted look at the psychodynamic effects of colonisation on the character and actions of Black Caribbeans, both in the islands and after migration to Europe. Influenced by Sartre's Anti-Semite and Jew, and reacting against Octave Mannoni's thesis that the colonial subject develops an inevitable psychological dependence on the coloniser, Fanon argued that West Indians had been stripped of their authenticity by first slavery and later imperialism. The French now proclaimed that the colonised, like Fanon himself, could become truly French (évolué) and take their place in the metropolis, but at the same time in subtle and not-so-subtle ways made it evident that this was quite impossible. Dramatically tracing the effect of racist stereotypes on daily life and on sexual and family relations, Fanon examines the European image of the Black as crudely physical, oversexed and intellectually impoverished, through using clinical case histories, memoirs and novels, government documents and psychoanalytical theories. The Creole response is 'lactification', a hopeless attempt to become as European as possible in education, taste and thought; just as the Whites become transformed through their own racism.

Frantz Fanon's argument and style influenced me as a junior psychiatrist in my first book Aliens and Alienists written with Maurice Lipsedge, and later when as an anthropologist, I lived with a new Trinidadian religion for a couple of years: away from an ethnic essentialism, he showed me how conflicting groups can mutually constitute each other in a perverse embrace under a political power which is generally held by one side alone. We are all creolised.

What is the relevance of Fanon today? Europe's empires have apparently fallen but in our globalised (a.k.a. neoliberal) world domination and power have hardly shifted their focus: the colonised are now more likely to be located in the metropole as well as in the global marketplace, while they still seek their identity through simulacra of the oppressors; as we, the beneficiaries, pursue our phantasmal desires at their expense. I particularly respond to his comments on the French colonial attempt to divest Algerian women of the haik (veil): yes, said Fanon, but not yet, and not at the bequest of the European - but by themselves, in their own time, and at their own wish. 